\title{
Evaluation of periostin level for predicting severity and chronicity of childhood atopic dermatitis
}

\author{
Deniz Ozceker ${ }^{1}$, Esra Yucel ${ }^{1}$, Sevgi Sipahi², Fatih Dilek³ ${ }^{3}$ Emin Ozkaya ${ }^{3}$, Eray Metin Guler ${ }^{4}$, Abdurrahim Kocyigit ${ }^{4}$, \\ Nermin Guler², Zeynep Tamay²
}

1Department of Pediatric Allergy and Immunology, Okmeydanı Education and Resarch Hospital, Saglik Bilimleri University, Istanbul, Turkey

${ }^{2}$ Department of Pediatric Allergy and Immunology, Istanbul Medical Faculty, Istanbul, Turkey

${ }^{3}$ Department of Pediatric Allergy and Immunology, Bezmi Alem University, Medical Faculty, Istanbul, Turkey

${ }^{4}$ Department of Biochemistry, Bezmi Alem University, Medical Faculty, Istanbul, Turkey

Adv Dermatol Allergol 2019; XXXVI (5): 616-619

DOI: https://doi.org/10.5114/ada.2018.79728

\begin{abstract}
Introduction: Periostin has some effects on the pathogenesis of atopic dermatitis (AD) via release of pro-inflammatory cytokines and chemokines from activated keratinocytes and it is related to chronicity of skin lesions.

Aim: To evaluate the relationship between plasma periostin levels and severity and chronicity of AD in children. Material and methods: The study population consisted of 29 children with atopic dermatitis without concomitant allergic disease such as asthma or allergic rhinitis and 31 healthy controls. Data of demographic features, serum eosinophil, total IgE and skin prick test results were collected through the patient's medical records. The severity of the disease was assessed by the SCORAD index. Serum periostin levels were measured with a human periostin ELISA kit.

Results: The mean ages of the AD patients and the control group participants were $80.7 \pm 52.8$ and $90.3 \pm 41.6$ months, respectively. Mean plasma periostin levels were $63.0 \pm 19.0 \mathrm{ng} / \mathrm{ml}$ in AD patients, and $23.6 \pm 7.3$ in healthy controls, and there was a statistically significant difference between the two groups $(p=0.001)$. Plasma periostin level did not vary according to total IgE or serum eosinophil count $(p>0.05)$. Age of onset and duration of symptoms also were not correlated with plasma periostin levels. Although there was a positive relationship between plasma periostin level and the SCORAD index of patients, it was not statistically significant $(r=0.19, p>0.05)$.

Conclusions: This study showed that plasma periostin levels were increased in children with atopic dermatitis. Periostin may have a partial role in the pathogenesis of atopic dermatitis, but it is not associated with severity or chronicity in children with atopic dermatitis.
\end{abstract}

Key words: atopic dermatitis, periostin, eczema, children.

\section{Introduction}

Periostin is an extracellular matrix (ECM) protein that belongs to the fasciclin family [1]. Periostin is expressed during ECM remodeling, mechanical stress, and postinjury tissue repair [2]. Many studies have revealed that periostin plays a role in various conditions such as allergic inflammation [3], cancer [4], fibro-proliferative disorders [5], myocardial infarction [6], and wound healing [7].

Atopic dermatitis (AD) is a chronic, pruritic, and relapsing inflammatory skin disease. It is accompanied by skin barrier dysfunction and dominant Th2 type inflammation $[8,9]$. It was recently found that periostin plays an important role in amplifying allergic skin inflammation by inducing Th2-type cytokines such as IL-4 and IL-13, which are related to the immune response [10, 11]. Additionally, periostin has some effects on the pathogenesis of $A D$ via the release of proinflammatory cytokines and chemokines such as thymic stromal lymphopoietin (TSLP), IL-25, and IL-33 from activated keratinocytes [12]. Further, it is related to the chronicity of skin lesions [13].

\section{Aim}

The purpose of this study was to evaluate the serum periostin level and its association with the severity and chronicity of $A D$ in children.

Address for correspondence: Deniz Ozceker MD, Department of Pediatric Allergy and Immunology, Okmeydanı Education and Resarch Hospital, Saglik Bilimleri University, 34100 Istanbul, Turkey, phone: +90 05435048856, e-mail: denizozceker@gmail.com Received: 19.09 .2018 , accepted: 15.10 .2018$. 


\section{Material and methods}

The study population consisted of 29 children with atopic dermatitis and 31 healthy children (control group) who were monitored at the Pediatric Allergy and Immunology outpatient clinics in the Saglık Bilimleri University Okmeydani Research and Training Hospital and Istanbul University, Istanbul Faculty of Medicine. The diagnosis of atopic dermatitis was made by a physician according to the Hanifin-Rajka criteria [14]. Children with atopic dermatitis had no concomitant allergic diseases such as asthma or allergic rhinitis. Data on demographic features such as age, gender, family history of atopy, age of onset and duration of symptoms, laboratory values of serum eosinophil, and total lgE and skin prick test results were collected through the patients' medical records. The severity of the disease was assessed by the SCORing Atopic Dermatitis (SCORAD) index [15].

\section{Laboratory investigation}

Venous blood samples from all patients were collected by antecubital venipuncture and dispensed directly into K3-EDTA tubes. The plasma separation was performed after centrifugation of the EDTA whole blood samples at $1500 \mathrm{~g}$ for $10 \mathrm{~min}$. The plasma specimens were kept frozen at $-80^{\circ} \mathrm{C}$ while they awaited analysis.

\section{Biochemical analysis}

The serum periostin levels were measured with the Human Periostin ELISA kit from Bioassay Technology Laboratory (Shanghai, China) according to the manufacturer's instructions.

\section{Skin prick tests}

The commercial allergen solutions manufactured by Stallergenes (Paris, France) were used for the skin prick tests. Ten different aeroallergens were applied in the skin prick tests: Alternaria, Aspergillus mixture, birch, cat epithelia, cypress, Dermatophagoides farinae, Dermatophagoides pteronyssinus, dog epithelia, grass pollen mixture, and weed pollen mixture. There were also six different food allergens: cocoa, egg white, egg yolk, milk, peanut, and wheat. The skin prick tests were considered positive if, after subtraction of the negative value, a wheal with a maximum diameter of at least three millimeters was present.

\section{Ethical approval}

The study was performed in accordance with good clinical practice and the tenets of the Declaration of Helsinki, and it was approved by the Ethical Committee of Istanbul Faculty of Medicine (2017/430). All study patients and their parents were given information about the study, and signed consent was obtained from the parents.

\section{Statistical analysis}

Statistical analyses were performed using IBM SPSS Statistics 19 (IBM, Armonk, NY, USA). The Shapiro-Wilk test was used to test distributions for normality. Parametric data are expressed as the mean \pm standard deviation (SD), and non-parametric data are expressed as the median and interquartile range (IQR). The Mann-Whitney $U$ test or Student's $t$-test was used to calculate the differences in variables between groups. The correlation between 2 variables was assessed using the Spearman rank correlation coefficient or Spearman's test. Categorical data were evaluated using the $\chi^{2}$ test; $p<0.05$ was accepted as statistically significant.

Table 1. Demographic and clinical values of patients and controls

\begin{tabular}{|c|c|c|c|}
\hline Parameter & $\begin{array}{l}\text { AD patients } \\
\quad(n=29)\end{array}$ & $\begin{array}{l}\text { Control group } \\
\qquad(n=31)\end{array}$ & $P$-value \\
\hline Age, mean \pm SD [months] & $80.7 \pm 52.8$ & $90.3 \pm 41.6$ & $>0.05$ \\
\hline Gender, M/F & $15 / 14$ & $18 / 13$ & $>0.05$ \\
\hline $\mathrm{SCORAD}$, mean $\pm \mathrm{SD}$ & $35.1 \pm 12.6$ & - & \\
\hline Serum total IgE > $100 \mathrm{IU} / \mathrm{I}, n(\%)$ & $34(58)$ & ND & \\
\hline Eosinophils > 4\%, $n(\%)$ & $18(62)$ & ND & \\
\hline Atopy, $n(\%)^{*}$ & $17(59)$ & ND & \\
\hline Food allergy, $n(\%)$ & $3(10)$ & - & \\
\hline Aeroallergen sensitivity, $n$ (\%) & $9(31 \%)$ & ND & \\
\hline \multicolumn{4}{|l|}{ Treatment, $n(\%)$ : } \\
\hline Emollient or moisturizer only & $9(32)$ & - & \\
\hline Topical steroid + emollient or moisturizer & $18(62)$ & & \\
\hline Topical calcineurin inhibitors + emollient or moisturizer & $2(7)$ & & \\
\hline
\end{tabular}




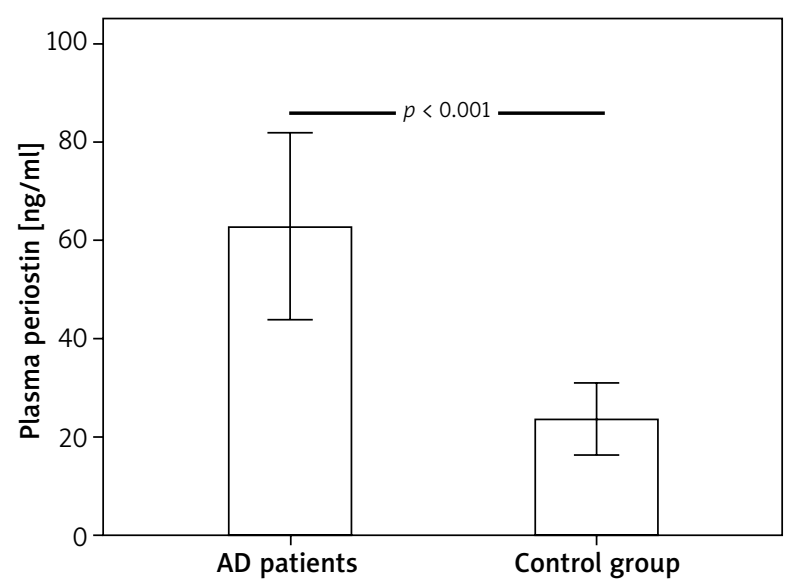

Figure 1. Serum periostin levels in study and control groups

\section{Results}

The study group consisted of 15 boys and 14 girls with $A D$, and the control group consisted of 18 boys and 13 girls. The mean ages (in months) of the AD patients and the control group participants were $80.7 \pm 52.8$ and $90.3 \pm 41.6$, respectively. No significant differences in age or gender existed between the groups ( $p>0.05)$. Some demographic and clinical features of the patients are shown in Table 1.

The mean plasma periostin levels were $63.0 \pm 19.0$ $\mathrm{ng} / \mathrm{ml}$ in the AD patients and $23.6 \pm 7.3$ in the control patients. There was a statistically significant difference between the two groups ( $p=0.001$ ) (Figure 1). The plasma periostin levels did not vary according to gender and atopy. Additionally, the plasma periostin levels did not correlate with the serum total IgE or the serum eosinophil count $(p>0.05)$. Additionally, the age of onset and duration of symptoms did not correlate with the plasma periostin levels (data not shown). Although there was a positive relationship between the plasma periostin levels and the SCORAD index of patients, it was not statistically significant $(r=0.19, p>0.05)$ (Figure 2).

\section{Discussion}

Atopic dermatitis is the most common inflammatory dermatological disorder. It is a chronic or chronically relapsing disease, and it is characterized by intense pruritus and dry skin [16]. Patients with AD have defective skin barrier functions and suffer increased transepithelial water loss. Dryness, when coupled with itching or scratching behaviors, is one of the main triggers of lichenification, which consists of epidermal hyperplasia and fibrosis [9]. Periostin, an ECM protein, was recently found to have a role in amplifying and maintaining skin inflammation

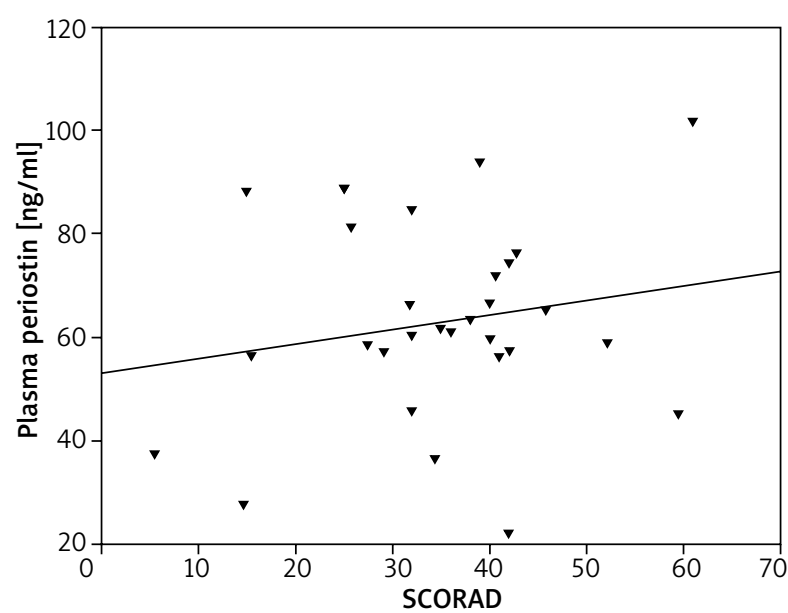

Figure 2. Scatter graph showing the plasma periostin levels and SCORAD relationship in patients with atopic dermatitis

by keratinocyte activation, along with increasing the Th2type immune response [17].

Increased levels of IL-13 in chronic skin lesions indicate its possible role in the proliferation and differentiation of keratinocytes and remodeling in AD. Similarly, IL-4 and IL-13-induced periostin production from airway epithelial cells reflects refractory eosinophilic inflammation and remodeling in asthma patients [18]. Using a mouse model of skin inflammation, Masuoka et al. demonstrated that periostin is a critical mediator for the amplification and persistence of allergic differentiation and proliferation of keratinocytes. Furthermore, Masuoka et al. also observed increased expression of periostin in the skin tissues of $A D$ patients and its correlation with AD severity [12]. Data derived from a recent study performed on canine AD (CAD) suggested that IL-13, possibly derived from T helper 2 (Th2) cells, stimulates periostin production in both keratinocytes and fibroblasts. This study has been accepted as a spontaneous atopic animal model. The authors commented that periostin may play a role in the enhancement and chronicity of skin lesions via IL-25 [13].

In the present study, we determined that plasma periostin levels were significantly higher in children with AD than in healthy children. Previous studies also determined that children and adults with AD had increased serum periostin levels [9-11]. Correlations between plasma periostin levels and serum total lgE and blood eosinophil levels have been reported in some studies [9]. However, we did not find a relationship between plasma periostin level and serum total IgE and blood eosinophil level. This may be due to the non-specificity of IgE and eosinophils in allergic diseases.

Recent studies suggested that plasma periostin levels may have a role in the severity and chronicity of AD. Sung et al. found a significant relationship between the SCORAD index and plasma periostin levels [19]. How- 
ever, their study group consisted primarily of patients with mild AD. In our study, there was no significant relationship between plasma periostin levels and severity of atopic dermatitis based on the SCORAD index. We think that the reason for this difference in the results is due to heterogeneity and the small sample size of the patients. For this reason, we cannot state a firm conclusion concerning this matter. The authors also reported higher plasma periostin levels in children with early onset of $A D$ ( $<2$ years of age) compared to those with an AD onset age under 2 years. Based on this finding, the investigators suggested that serum periostin can be a biomarker of AD chronicity. We did not find any significant relationship between the plasma periostin levels and age at $A D$ onset. Kou et al. [20] detected higher levels of serum periostin in patients with erythrodermic type AD and widespread type $A D$, as compared to patients with lesions that had not spread systemically. Uysal et al. reported significant correlations between plasma periostin levels and the duration of symptoms and the presence of atopy [21]. Our results contrast with these study results, and this difference may be associated with sample sizes, recruitment strategies and ELISA kit-specific changes.

As it has with asthma, biomarker-based endotyping in AD has engendered new hope for predicting prognoses and improved therapy outcomes. Various biomarkers such as TARC, TSLP, IL-31, and IL-33 have been investigated to evaluate the pathogenesis of AD and to predict severity and chronicity. TARC and TLSP were found to be related to AD severity and chronicity [21]. Our study has some limitations. The research was conducted with a small group of patients, and, unlike patients in other studies, ours were more likely to have moderate to severe atopic dermatitis.

\section{Conclusions}

Plasma periostin levels are higher in patients with atopic dermatitis. Periostin may play a partial role in the pathogenesis of atopic dermatitis. We cannot determine the potential of serum periostin as an indicator of the severity and chronicity of atopic dermatitis in childhood in contrast to previously published studies.

\section{Conflict of interest}

The authors declare no conflict of interest.

\section{References}

1. Horiuchi K, Amizuka N, Takeshita S, et al. Identification and characterization of a novel protein, periostin, with restricted expression to periosteum and periodontal ligament and increased expression by transforming growth factor beta. J Bone Miner Res 1999; 14: 1239-49.

2. Sidhu SS, Yuan S, Innes AL, et al. Roles of epithelial cell-derived periostin in TGF-beta activation, collagen production, and collagen gel elasticity in asthma. Proc Natl Acad Sci USA 2010; 107: 14170-5.

3. Takayama G, Arima K, Kanaji T, et al. Periostin: a novel component of subepithellial fibrosis of bronchial asthma downstream of IL-4 and IL-13 signals. J Allergy Clin Immunol 2006; 118: 98-104.

4. Jin J, Son M, Kim H, et al. Comparative proteomic analysis of human malignant ascitic fluids for the development of gastric cancer biomarkers. Clin Biochem 2018; 56: 55-61.

5. Heo SC, Shin WC, Lee MJ, et al. Periostin accelerates bone healing mediated by human mesenchymal stem cell-embedded hydroxyapatite/tricalcium phosphate scaffold. PLoS One 2015; 10: e0116698.

6. Eschenhagen T. A new concept of fibroblast dynamics in post-myocardial infarction remodeling. J Clin Invest 2018; 128: 1731-3.

7. Walker JT, McLeod K, Kim S, et al. Periostin as a multifunctional modulator of the wound healing response. Cell Tissue Res 2016; 365: 453-65.

8. Brunner PM, Guttman-Yassky E, Leung DY. The immunology of atopic dermatitis and its reversibility with broad-spectrum and targeted therapies. J Allergy Clin Immunol 2017; 139: S65-76.

9. Kim BE, Leung DY. Epidermal barrier in atopic dermatitis. Allergy Asthma Immunol Res 2012; 4: 12-6.

10. Yamaguchi Y. Periostin in skin tissue and skin-related disease. Allergol Int 2014; 63: 161-70.

11. Oyoshi MK, He R, Kumar L, et al. Cellular and molecular mechanisms in atopic dermatitis. Adv Immunol 2009; 102: 135-226.

12. Masuoka M, Shiraishi H, Ohta S, et al. Periostin promotes chronic allergic inflammation in response to Th2 cytokines. J Clin Invest 2012; 122: 2590-600.

13. Mineshige T, Kamiie J, Sugahara G, Shirota K. A study on periostin involvement in the pathophysiology of canine atopic skin. J Vet Med Sci 2018; 80: 103-11.

14. Hanifin JM, Rajka G. Diagnostic features of atopic dermatitis. Acta Derm Venereol 1980; 92: 44-7.

15. Kunz B, Oranje AP, Labreze L, et al. Clinical validation and guidelines for the SCORAD index: consensus report of the European task force on atopic dermatitis. Dermatology 1997; 195: 10-9.

16. Weidinger S, Novak N. Atopic dermatitis. Lancet 2016; 387: 1109-22.

17. Shiraishi H, Masuoka M, Ohta S, et al. Periostin contributes to the pathogenesis of atopic dermatitis by inducing TSLP production from keratinocytes. Allergol Int 2012; 61: 563-72.

18. Matsumo H. Serum periostin: a novel biomarker for asthma management. Allergol Int 2014; 63: 153-60.

19. Sung M, Lee KS, Ha EG, et al. An association of periostin levels with the severity and chronicity of atopic dermatitis in children. Pediatr Allergy Immunol 2017; 28: 543-50.

20. Kou K, Okawa T, Yamaguchi Y, et al. Periostin levels correlate with disease severity and chronicity in patients with atopic dermatitis. Br J Dermatol 2014; 171: 283-91.

21. Uysal P, Birtekocak F, Karul AB. The relationship between serum TARC, TSLP and POSTN levels and childhood atopic dermatitis. Clin Lab 2017; 63: 1071-7. 